\title{
Overluminous HNC line emission in Arp 220, NGC 4418 and Mrk 231. Global IR pumping or XDRs?
}

\author{
S. Aalto ${ }^{1}$, M. Spaans ${ }^{2}$, M. C. Wiedner ${ }^{3}$, and S. Hüttemeister ${ }^{4}$ \\ 1 Onsala Rymdobservatorium, Chalmers Tekniska Högskola, 43992 Onsala, Sweden \\ e-mail: susanne@oso.chalmers.se \\ 2 Kapteyn Astronomical Institute, PO Box 800, 9700 AV Groningen, The Netherlands \\ 3 I. Physikalisches Institut, Universität zu Köln, Zülpicher Str. 77, 50937 Köln, Germany \\ 4 Astronomisches Institut der Universität Bochum, Universitätsstraße 150, 44780 Bochum, Germany
}

Received 29 September 2006 / Accepted 29 November 2006

\section{ABSTRACT}

\begin{abstract}
Context. In recent studies of $3 \mathrm{~mm} J=1-0 \mathrm{HNC}$ emission from galaxies it is found that the emission is often bright which is unexpected in warm, star forming clouds. We propose that the main cause for the luminous HNC line emission is the extreme radiative and kinematical environment in starburst and active nuclei.

Aims. To determine the underlying excitational and chemical causes behind the luminous HNC emission in active galaxies and to establish how HNC emission may serve to identify important properties of the nuclear source.

Methods. We present mm and submm JCMT, IRAM $30 \mathrm{~m}$ and CSO observations of the $J=3-2$ line of HNC and its isomer HCN in three luminous galaxies and $J=4-3 \mathrm{HNC}$ observations of one galaxy. The observations are discussed in terms of physical conditions and excitation as well as in the context of X-ray influenced chemistry.

Results. The ultraluminous mergers Arp 220 and Mrk 231 and the luminous IR galaxy NGC 4418 show the HNC $J$ 3-2 emission being brighter than the HCN 3-2 emission by factors of 1.5 to 2. We furthermore report the detection of HNC $J=4-3$ in Mrk 231 . Overluminous $\mathrm{HNC}$ emission is unexpected in warm molecular gas in ultraluminous galaxies since $I(\mathrm{HNC}) \gtrsim I(\mathrm{HCN})$ is usually taken as a signature of cold $(10-20 \mathrm{~K})$ dark clouds. Since the molecular gas of the studied galaxies is warm $\left(T_{\mathrm{k}} \gtrsim 40 \mathrm{~K}\right)$, we present two alternative explanations to the overluminous HNC: a) HNC excitation is affected by pumping of the rotational levels through the mid-infrared continuum and b) XDRs (X-ray Dominated Regions) influence the abundances of HNC.

$\mathrm{HNC}$ may become pumped at $21.5 \mu \mathrm{m}$ brightness temperatures of $T_{\mathrm{B}} \gtrsim 50 \mathrm{~K}$, suggesting that $\mathrm{HNC}$-pumping could be common in warm, ultraluminous galaxies with compact IR-nuclei. This means that the HNC emission is no longer dominated by collisions and its luminosity may not be used to deduce information on gas density. On the other hand, all three galaxies are either suspected of having buried AGN - or the presence of AGN is clear (Mrk 231) - indicating that X-rays may affect the ISM chemistry.

Conclusions. We conclude that both the pumping and XDR alternatives imply molecular cloud ensembles distinctly different from those of typical starforming regions in the Galaxy, or the ISM of less extreme starburst galaxies. The HNC molecule shows the potential of becoming an additional important tracer of extreme nuclear environments.
\end{abstract}

Key words. galaxies: evolution - galaxies: general - galaxies: starburst - galaxies: active - radio lines: galaxies - ISM: molecules

\section{Introduction}

In order to understand the AGN and star formation activity in the centres of luminous galaxies it is essential to study the prevailing conditions of the dense $\left(n\left(\mathrm{H}_{2}\right) \geq 10^{4} \mathrm{~cm}^{-3}\right)$ molecular gas. The polar molecule HCN (dipole moment 2.98 debye) is commonly used as a tracer of this dense gas-phase. In particular in distant luminous $\left(L_{\mathrm{IR}}>10^{11} L_{\odot}\right.$, LIRGs) and ultraluminous ( $L_{\mathrm{IR}}>10^{12} L_{\odot}$, ULIRGs) systems the HCN $1-0$ line is the prototypical tracer of dense gas content (e.g. Solomon et al. 1992; Helfer \& Blitz 1993; Curran et al. 2000; Gao \& Solomon 2004). $\mathrm{HNC}$, the isomer of $\mathrm{HCN}$, traces gas of equally high density. In dense, Galactic, molecular cloud cores it has been suggested to trace gas temperature: Neutral-neutral chemical models predict that the $\mathrm{HCN} / \mathrm{HNC}$ abundance ratio increases with increasing temperature. This is supported by the fact that the measured HCN abundance ratio is especially high in the vicinity of the hot core of Orion KL (e.g. Schilke et al. 1992; Hirota et al. 1998).

It is therefore surprising that the $H N C / H C N J=1-0$ intensity ratios are found to be low in luminous galaxies (e.g.
Hüttemeister et al. 1995; Aalto et al. 2002 (APHCO2)) and that the HNC/HCN line ratio appears to increase with galactic luminosity (APHCO2).

As an explanation to the abnormally bright HNC emission, APHC02 suggest that the processes are dominated by fast ionneutral chemistry in moderately dense PDR-like regions, instead of the neutral-neutral chemistry likely governing the hot dense cores of the Orion cloud. In this case, one would expect the $\mathrm{HCN}$ and HNC abundances and excitation to be very similar to each other - independently of gas temperature. A study of the higher rotational transitions should show equal brightness for the two species.

Another possible scenario suggested by APHC02 is that, instead of being collisionally excited, HNC is being radiatively excited. HNC may be pumped by $21.5 \mu \mathrm{m}$ continuum radiation through vibrational transitions in its degenerate bending mode. This would likely result in significant differences in the HCN and HNC excitation.

Alternatively, Meijerink \& Spaans (2005) suggest that the $\mathrm{HNC}$ abundance may become enhanced over that of $\mathrm{HCN}$ in 


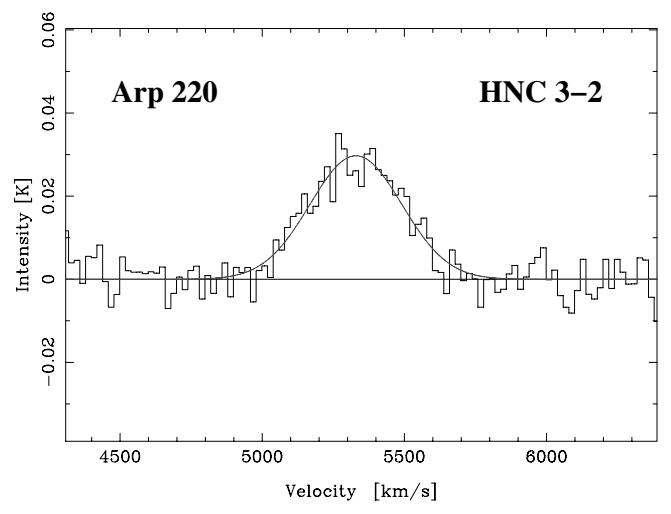

Fig. 1. JCMT HNC 3-2 spectrum of Arp 220. The scale is in $T_{\mathrm{A}}^{*}$ and should be multiplied with $1 / 0.69=1.45$ for the scale to be transferred into main beam brightness (see Table 1). The velocity resolution has been smoothed to $30 \mathrm{~km} \mathrm{~s}^{-1}$. The Gaussian fit is presented in Table 3 .

warm, dense XDRs (X-ray Dominated Regions). The deeply penetrating $\mathrm{X}$-rays induce an ionization structure that differs from the one in a PDR and that favors asymmetries which exist in the HNC and HCN chemical pathways. This offers a chemical explanation for the abnormal HNC luminosity that is linked to the accreting black hole.

In order to investigate the underlying cause behind the bright HNC emission in luminous galaxies, we have searched for HCN and HNC $J=3-2$ emission in a sample of LIRG and ULIRG galaxies with the JCMT and IRAM $30 \mathrm{~m}$ telescopes. This will allow us to investigate the excitation of the HNC and HCN molecules. In this paper, we present the first results of this study on the HNC 3-2 emission of two ultralumious galaxies, Arp 220 and Mrk 231, and one luminous IR galaxy, NGC 4418. For these galaxies we find the HNC 3-2 luminosity to be greater than that of $\mathrm{HCN} J=3-2$ and we discuss two possible scenarios: mid-infrared (mid-IR) pumping and X-ray dominated chemistry (XDRs). From now on, we omit the $J$ and refer to a rotational transition only as: 3-2 instead of: $J=3-2$.

\section{Observations and results}

We have used the JCMT telescope to measure the HNC 3-2 (271 GHz) (Figs. 1 and 2) lines towards the ultraluminous galaxies Arp 220 and Mrk 231. Observations were made in April 2005, and the system temperatures were typically $450 \mathrm{~K}$. Pointing was checked regularly on $\mathrm{SiO}$ masers and the rms was found to be 2 ". Furthermore, the HNC 4-3 line ( $353 \mathrm{GHz})$ was observed in Mrk 231 in February 2006. In addition, we observed the HCN 3-2 (Fig. 3) line in Mrk 231 with the CSO telescope in 1997. With JCMT we also observed the ${ }^{12} \mathrm{CO} 2-1$ line of Mrk 231 and used it to compare with the same line observed with the CSO towards the same galaxy to compare the intensity scales of the two telescopes. The ${ }^{12} \mathrm{CO} 2-1$ line intensities from the two telescopes were found to agree within $10 \%$. The HNC and HCN 3-2 lines of NGC 4418 were observed in May and July 2006 with the IRAM 30 telescope. Pointing rms ranged between 1.5 and 2 and system temperatures ranged between 400 and $600 \mathrm{~K}$. Beam sizes and efficiencies are shown in Table 1. Molecular line ratios are presented in Table 2. Note that line intensities were corrected for beam size when compared to each other.
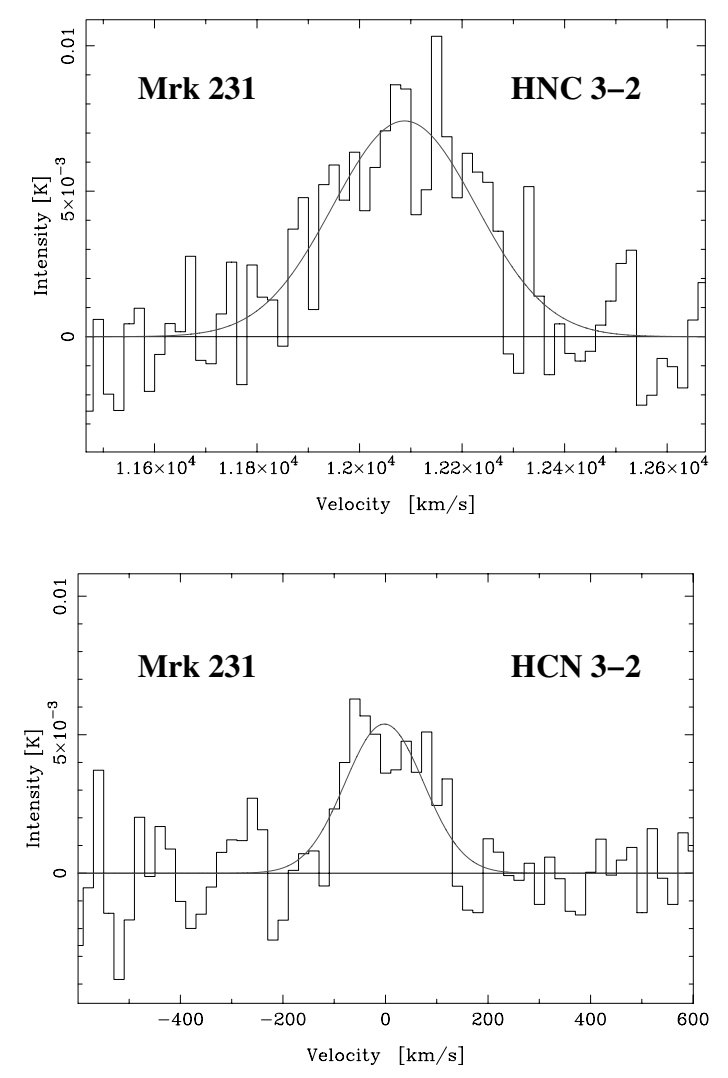

Fig. 2. Upper panel: JCMT HNC 3-2 spectrum of Mrk 231. The scale is in $T_{\mathrm{A}}^{*}$ and should be multiplied with $1 / 0.7=1.43$ for the scale to be transferred into main beam brightness (see Table 1). The velocity resolution has been smoothed to $20 \mathrm{~km} \mathrm{~s}^{-1}$. Lower panel: CSO HCN 3-2 spectrum of Mrk 231. The scale is in $T_{\mathrm{A}}^{*}$ and should be multiplied with $1 / 0.69$ for the scale to be transferred into main beam brightness (see Table 1). The velocity resolution has been smoothed to $20 \mathrm{~km} \mathrm{~s}^{-1}$. The velocity scales are different due to two different observing approaches. The spectra, however, have been scaled to the same resolution and bandwidth so that the different line widths are apparent. The total available bandwidth for the HNC spectrum is not shown here to enable comparison with the HCN spectrum. The Gaussian fits are presented in Table 3.

\section{1. $\operatorname{Arp} 220$}

The HNC 3-2 line is about a factor of two brighter than the HCN 3-2 line (see Table 2). For Arp 220, we find that the HNC excitation is superthermal with a $3-2 / 1-0$ ratio of 1.8 . Wiedner et al. (2004) find the HCN 3-2/1-0 ratio to be thermal with ratios close to 0.9 (see Table 2). The HCN and HNC line widths are similar although there is a difference in line shape.

\section{2. $M r k 231$}

In Mrk 231, the HNC 3-2 line is a factor of 1.5 brighter than the HCN 3-2 line (Table 2). In contrast to Arp 220 this is mainly due to the larger line width of the HNC line, while the intensities for the two lines (corrected for the difference in beam size) is similar (Table 3 ). The HNC $3-2 / 1-0$ ratio is about 0.7 while the HCN 3-2/1-0 ratio is 0.3 (Table 2). The $\mathrm{HNC} / \mathrm{HCN}$ intensity ratio is close to unity in the $1-0$ transition (APHC02). The HNC 4-3 line is clearly detected and the HNC 4-3/3-2 ratio is 0.5 , but the lack of available baseline renders the estimated integrated intensity somewhat uncertain. 


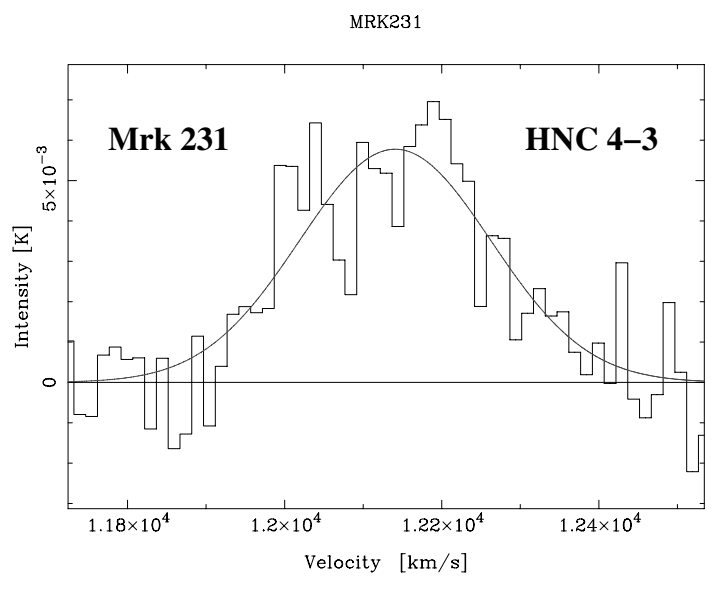

Fig. 3. JCMT HNC 4-3 spectrum of Mrk 231. The scale is in $T_{\mathrm{A}}^{*}$ and should be multiplied with $1 / 0.63=1.6$ for the scale to be transferred into main beam brightness (see Table 1). The velocity resolution has been smoothed to $20 \mathrm{~km} \mathrm{~s}^{-1}$. The Gaussian fit is presented in Table 3 .

Table 1. Observational parameters.

\begin{tabular}{lccc}
\hline \hline Transition & $v[\mathrm{GHz}]$ & $H P B W\left[{ }^{\prime \prime}\right]$ & $\eta_{\mathrm{mb}}$ \\
\hline JCMT: & & & \\
HCN 3-2 & 266 & 20 & 0.69 \\
HNC 3-2 & 271 & 19 & 0.69 \\
HNC 4-3 & 353 & 14 & 0.63 \\
CSO: & & & \\
HCN 3-2 & 267 & 30 & 0.69 \\
CO 2-1 & 230 & 34 & 0.69 \\
IRAM: & & & \\
HCN 3-2 & 267 & 9.5 & 0.46 \\
HNC 3-2 & 271 & 9.5 & 0.46 \\
\hline
\end{tabular}

Table 2. Line ratios.

\begin{tabular}{lccc}
\hline \hline Galaxy & $\frac{\text { HNC }}{\text { HCN }} 3-2$ & HNC $\frac{3-2}{1-0}$ & HCN $\frac{3-2}{1-0}$ \\
\hline Arp 220 & $1.9 \pm 0.3^{a}$ & $1.8 \pm 0.3^{b}$ & $0.9^{c}$ \\
Mrk 231 & $1.5 \pm 0.2$ & $0.7 \pm 0.2^{b}$ & $0.3 \pm 0.1^{d}$ \\
NGC 4418 & $2.3 \pm 0.3$ & $0.8 \pm 0.2^{e}$ & $0.3 \pm 0.1$ \\
\hline
\end{tabular}

a The HNC/HCN 3-2 line ratio is consistent with the one found by Cernicharo et al. (2006); ${ }^{b} \mathrm{HNC} 1-0$ from APCH02; ${ }^{c} \mathrm{HCN} 3-2$ from Wiedner et al. (2004); ${ }^{d} \mathrm{HCN} 1-0$ data from Curran et al. (2000); ${ }^{e} \mathrm{HNC} 1-0$ data from Monje et al. (in preparation). HCN 1-0 from Kohno et al. (2004). The HNC 4-3/3-2 line ratio for Mrk 231 is 0.5 but the lack of baseline for the HNC 4-3 data renders this value somewhat uncertain. Stated errors are $2 \sigma$ rms errors.

\subsection{NGC 4418}

We find that the HNC 3-2 emission is brighter than the HCN emission by a factor of 2.3 (Table 2) which makes NGC 4418 the galaxy with the largest $\mathrm{HNC} / \mathrm{HCN} 3-2$ ratio measured so far. The difference in integrated intensity is due to the HNC line having a significantly higher intensity while its line width is narrower than that of HCN (Table 3). Furthermore, the excitation between $\mathrm{HCN}$ and $\mathrm{HNC}$ is distinctly different: while $\mathrm{HCN}$ is subthermally excited with $3-2 / 1-0$ line ratios of 0.3 , we find that the corresponding ratio for $\mathrm{HNC}$ is closer to unity.
Table 3. Gaussian $\mathrm{HNC}$ and $\mathrm{HCN}$ line fits.

\begin{tabular}{lccc}
\hline \hline Transistion & Arp 220 & NGC 4418 & Mrk 231 \\
\hline $\int I\left(\mathrm{HNC} \mathrm{3-2}^{2}\right)\left[\mathrm{K} \mathrm{km} \mathrm{s}^{-1}\right]$ & $18.6 \pm 0.7^{a}$ & $10.8 \pm 1^{b}$ & $2.9 \pm 0.2^{a}$ \\
$V_{\mathrm{c}}\left[\mathrm{km} \mathrm{s}^{-1}\right]$ & 5330 & 2120 & 12095 \\
$\Delta V\left[\mathrm{~km} \mathrm{~s}^{-1}\right]$ & 390 & 150 & 313 \\
$T_{\text {peak }}[\mathrm{mK}]$ & 44 & 63 & 9.0 \\
\hline $\int I\left(\mathrm{HNC} \mathrm{4-3)}\left[\mathrm{K} \mathrm{km} \mathrm{s}^{-1}\right]\right.$ & - & - & $2.7 \pm 0.2^{a}$ \\
$V_{\mathrm{c}}\left[\mathrm{km} \mathrm{s}^{-1}\right]$ & - & - & 12144 \\
$\Delta V\left[\mathrm{~km} \mathrm{~s}^{-1}\right]$ & - & - & 278 \\
$T_{\text {peak }}[\mathrm{mK}]$ & - & - & 9.0 \\
\hline $\int I(\mathrm{HCN} 3-2)\left[\mathrm{K} \mathrm{km} \mathrm{s}^{-1}\right]$ & - & $5.2 \pm 0.6^{b}$ & $0.7 \pm 0.1^{c}$ \\
$V_{\mathrm{c}}\left[\mathrm{km} \mathrm{s}^{-1}\right]$ & - & 2120 & 12095 \\
$\Delta V\left[\mathrm{~km} \mathrm{~s}^{-1}\right]$ & - & 240 & 178 \\
$T_{\text {peak }}\left[\mathrm{mK}^{-1}\right.$ & - & 20 & 4.2 \\
\hline
\end{tabular}

${ }^{a}$ JCMT; ${ }^{b}$ IRAM $30 \mathrm{~m} ;{ }^{c}$ CSO. Integrated intensities and line intensities are in main beam brightness scale. Note that the data are taken with different beam sizes due to the different telescopes. This has been corrected for in the line ratio table.
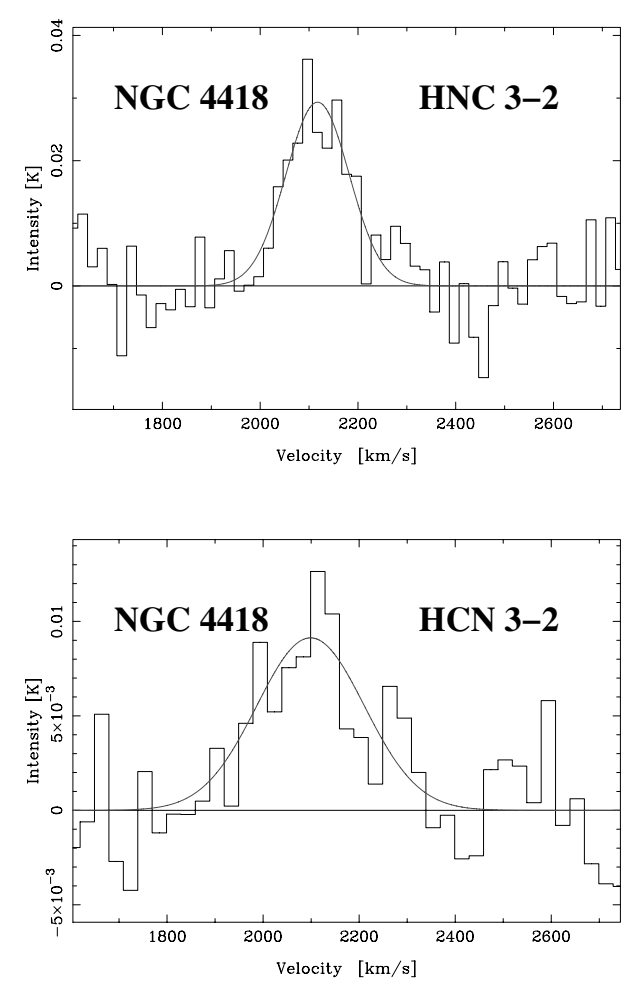

Fig. 4. IRAM HNC (upper panel) and HCN (lower panel) 3-2 spectrum of NGC 4418. The scale is in $T_{\mathrm{A}}^{*}$ and should be multiplied with $1 / 0.46=2.17$ for the scale to be transferred into main beam brightness (see Table 1). The velocity resolution has been smoothed to $30 \mathrm{~km} \mathrm{~s}^{-1}$. The Gaussian fit is presented in Table 3.

\section{The origin of overluminous HNC}

\subsection{HNC in Galactic molecular clouds}

In ion-neutral chemical processes $\mathrm{HCNH}^{+}$will recombine to produce either $\mathrm{HCN}$ or $\mathrm{HNC}$ with (roughly) $50 \%$ probability. In addition, the reaction $\mathrm{H}_{2} \mathrm{NC}^{+}+\mathrm{e} \rightarrow \mathrm{HNC}+\mathrm{H}$, is suggested to produce more HNC (e.g., Hirota et al. 1998). In the Milky Way, these reactions occur mainly in dark clouds for kinetic temperatures below $24 \mathrm{~K}$. In clouds with higher temperatures, the above reactions are replaced by neutral-neutral reactions which tend 
Table 4. Mid-IR and dust properties of Arp 220, NGC 4418 and Mrk 231

\begin{tabular}{lcccc}
\hline \hline Galaxy & $\begin{array}{c}L_{\mathrm{FIR}} \\
{\left[L_{\odot}\right]}\end{array}$ & $\begin{array}{c}A_{\mathrm{V}} \\
{[\mathrm{mag}]}\end{array}$ & $\begin{array}{c}T_{\mathrm{K}}(\text { gas }) \\
{[\mathrm{K}]}\end{array}$ & $\begin{array}{c}T_{\mathrm{D}}(\text { dust }) \\
{[\mathrm{K}]}\end{array}$ \\
\hline Arp 220 & $1.1 \times 10^{12 a}$ & $1000^{b}$ & $\gtrsim 40^{b}$ & $\gtrsim 85^{c, d}$ \\
NGC 4418 & $8 \times 10^{10 e}$ & $\gtrsim 50^{f}$ & - & $85^{g}$ \\
Mrk 231 & $2.3 \times 10^{12}$ & - & $>34^{h}$ & $128^{i}$ \\
\hline
\end{tabular}

${ }^{a}$ Aalto et al. (1991); ${ }^{b}$ Sakamoto et al. (1999); ${ }^{c}$ Soifer et al. (1999) find that the two nuclei of Arp 220 are probably optically thick at $24.5 \mu \mathrm{m}$; ${ }^{d}$ Gonzáles-Alfonso et al. (2004) have modelled the far-infrared spectrum of Arp 220 as a two-component model consisting of a nuclear region of $T=106 \mathrm{~K}$, effective size of $0 .^{\prime} 4$ and optically thick in the farinfrared - surrounded by an extended region of size $2^{\prime \prime} ;{ }^{e}$ Roche et al. (1986); ${ }^{f}$ Spoon et al. (2001); ${ }^{g}$ Evans et al. (2003) found that the midIR emitting source is compact and is consistent with a $70 \mathrm{pc}$ source of brightness temperature (dust temperature) $85 \mathrm{~K} ;{ }^{h}$ Bryant \& Scoville (1999); ${ }^{i}$ Soifer et al. (2000) find a compact source of radius less than $100 \mathrm{pc}$ at $12.5 \mu \mathrm{m}$ and with brightness temperature $T_{\mathrm{B}} \gtrsim 141 \mathrm{~K}$. If the IR emitting source has the same size at $25 \mu \mathrm{m}$, the corresponding brightness temperature is $128 \mathrm{~K}$.

to selectively destroy $\mathrm{HNC}$ resulting in $X[\mathrm{HNC}]<X[\mathrm{HCN}]$. In these neutral-neutral reactions $\mathrm{HNC}$ reacts with hydrogen and oxygen to form $\mathrm{HCN}, \mathrm{NH}$ and $\mathrm{CO}$. Thus, in Galactic, warm star forming regions, $\mathrm{HNC}$ is underabundant compared to $\mathrm{HCN}$ (e.g. Schilke et al. 1992).

\subsection{HNC in luminous galaxies}

In contrast to the findings for Galactic, warm starforming regions, APHC02 found significant HNC 1-0 luminosities in the centres of warm starburst galaxies suggesting that high abundances of $\mathrm{HNC}$ were produced in warm environments. APHCO2 suggested that this is due to ion-neutral reactions persisting despite high temperatures in the gas $(40-150 \mathrm{~K})$. The presence of PDRs (Photon Dominated Regions) could provide an environment where $X[\mathrm{HNC}]=X[\mathrm{HCN}]$ and the luminosities of the two species would be similar. In this paper, however, we report an overluminosity of HNC compared to HCN by factors of 1.5 to 2.3. The PDR chemistry alone does not offer a scenario where $I[H N C] \gtrsim I[H C N]$ and in the following two subsections we present two mechanisms that can reproduce the observed overluminosity of HNC: mid-IR pumping and XDR chemistry.

\subsection{IR pumping of HNC}

Both HCN and HNC have degenerate bending modes in the IR. The molecule absorbs IR-photons to the bending mode (its first vibrational state) and then it decays back to the ground state via its $P$ branch $(v=1-0, \Delta J=+1)$ or $R$-branch $(v=1-0$, $\Delta J=-1$ ) (see Fig. 5). In this way, a vibrational excitation may produce a change in the rotational state in the ground level and can be treated (effectively) as a collisional excitation in the statistical equations. Thus, IR pumping excites the molecule to the higher rotational level by a selection rule $\Delta J=2$.

For HNC, the bending mode occurs at $\lambda=21.5 \mu \mathrm{m}$ $\left(464.2 \mathrm{~cm}^{-1}\right)$ with an energy level $h v / k=669 \mathrm{~K}$ and an $A$-coefficient of $A_{\mathrm{IR}}=5.2 \mathrm{~s}^{-1}$. For HCN the mode occurs at $\lambda=14 \mu \mathrm{m}\left(713.5 \mathrm{~cm}^{-1}\right)$, energy level $h v / k=1027 \mathrm{~K}$ and $A_{\mathrm{IR}}=$ $1.7 \mathrm{~s}^{-1}$. It is therefore significantly easier to pump HNC, than $H C N$. The pumping of HNC may start to become effective when

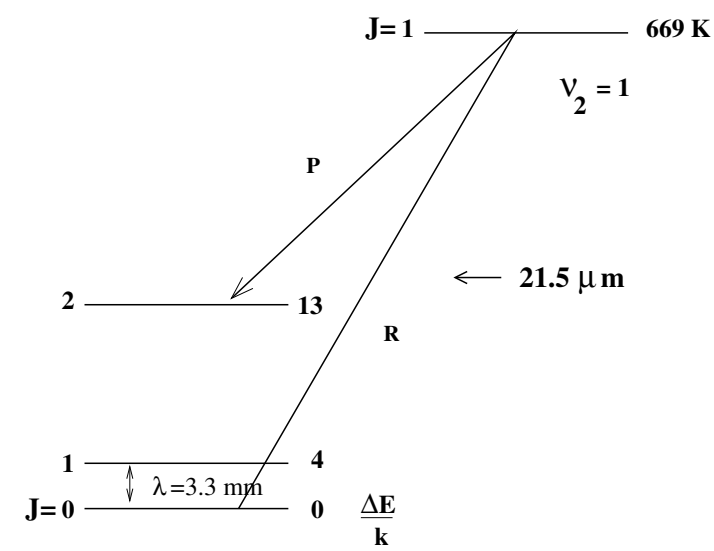

Fig. 5. A schematic picture of the pumping of the HNC rotational levels via the mid IR bending transitions. The figure shows how the rotational $J=2$ level may become populated through the $\Delta J=2$ selection rule through mid-IR pumping. The principle is the same for higher $J$ levels.

the IR background reaches an optically thick brightness temperature of $T_{\mathrm{B}} \approx 50 \mathrm{~K}$ and the gas densities are below critical.

The competition between collisions and radiative excitation

The IR pumping will compete with collisions for the excitation of $\mathrm{HNC}$ - when the IR field becomes more intense, higher densities are required to successfully compete with the radiative excitation. A simplified analysis includes comparing the rate of collisional excitation with the rate of mid-IR photon absorption. A wavelength of $21.5 \mu \mathrm{m}$ corresponds to a photon energy of $E / k=669 \mathrm{~K}$ and the IR pumping rate is roughly $P_{\mathrm{IR}} \propto A_{\mathrm{IR}} /\left(\mathrm{e}^{669 / T_{\mathrm{B}}}-1\right)$ where the $T_{\mathrm{B}}$ is the mid-IR brightness temperature (optically thick dust temperature) and $A_{\mathrm{IR}}$ is the Einstein coefficient for the mid-IR bending transition. For $\mathrm{HNC}$, the collisional rate is roughly $2 \times 10^{-10} n\left(\mathrm{H}_{2}\right) \mathrm{s}^{-1}$ where $n$ is in $\mathrm{cm}^{-3}$. $A_{\mathrm{IR}}$ for $\mathrm{HNC}$ is $5.2 \mathrm{~s}^{-1}$, so when $T_{\mathrm{B}}=55 \mathrm{~K}$, the pumping rate is $2.7 \times 10^{-5}$ may dominate at gas densities less than $10^{5} \mathrm{~cm}^{-3}$. When $T_{\mathrm{B}}$ is $85 \mathrm{~K}$, then the IR pumping rate is almost 100 times faster than at $55 \mathrm{~K}$. Radiative excitation through mid-IR pumping may then dominate over collisional excitation up to a density greater than $n=10^{6} \mathrm{~cm}^{-3}$. These rough numbers illustrate how sensitive the competition between pumping and collisions is to the background IR brightness.

For $\mathrm{HCN}$, the pump rate is two orders of magnitude slower at $85 \mathrm{~K}$, compared to HNC. This is because the $A_{\mathrm{IR}}$ coefficient is lower and the energy level for the bending mode is higher (see previous section).

\section{Pumping in a two-phase molecular medium}

In a situation where the pumping completely dominates the excitation of HNC the excitation temperatures of the rotational levels approach that of the brightness temperature of the background IR field. However, in reality the scenario is unlikely to be this simple. The molecular ISM will, for example, consist of a range of densities - also in the nucleus of the galaxy.

Consider a simple molecular ISM where the dense $(n \gtrsim$ $10^{4} \mathrm{~cm}^{-3}$ ) cores in the galaxy nuclei are surrounded by lower density $\left(n=10^{2}-10^{3} \mathrm{~cm}^{-3}\right)$, unbound gas (a "raisin roll" scenario - e.g. Aalto et al. 1995; Downes \& Solomon 1998; Hüttemeister \& Aalto 2001; Aalto 2005). In this scenario, the diffuse gas contributes a significant fraction of the lower- $J{ }^{12} \mathrm{CO}$ emission and has a high volume filling factor. In the case of an intense mid-IR field, all of the HNC molecules exposed to it will be dominated by the radiative excitation. In a more dilute field, with $T_{\mathrm{B}}=50 \mathrm{~K}$, for example, the dense cores may be unaffected while the HNC molecules in the more diffuse gas may be 

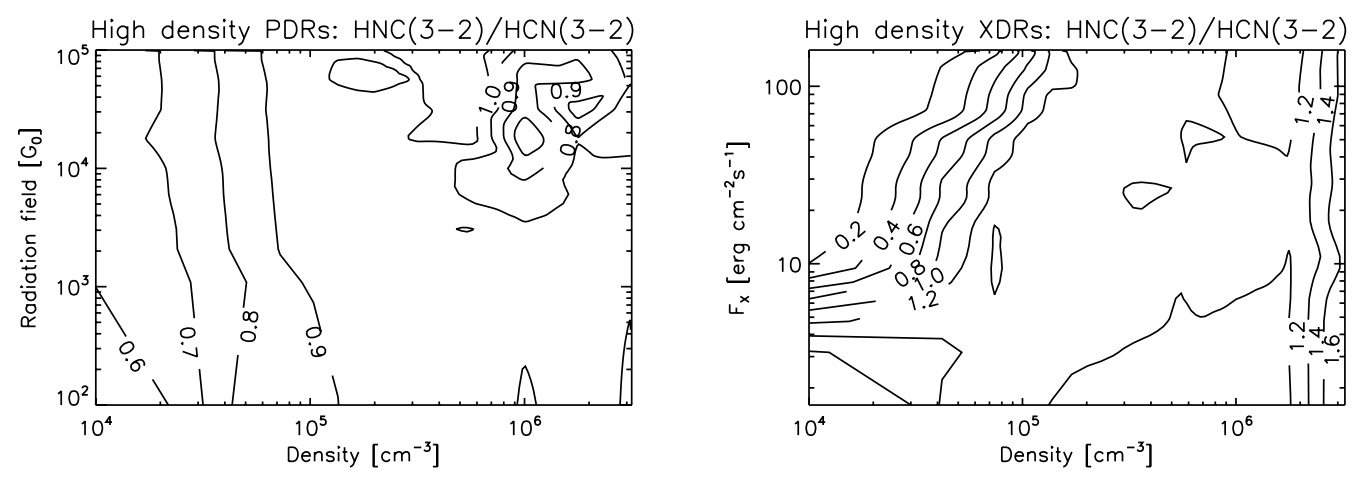

Fig. 6. Model line ratio HNC/HCN 3-2: PDRs (left panel) and XDRs (right panel). Note that PDR models cannot reproduce ratios larger than unity, whereas XDRs can. All models are for a 1 pc cloud.

experiencing radiative excitation. Thus, the global HNC luminosity may be significantly affected by pumping, even if the mid$I R$ field is moderate. In this scenario one would expect a radial gradient where the effect of the radiative pumping affects a larger fraction of the gas closer to the nucleus of the galaxy.

The presence of such a diffuse, lower density $(n=$ $10^{2}-10^{3} \mathrm{~cm}^{-3}$ ) molecular medium is suggested to be present in Arp 220: a large ${ }^{12} \mathrm{CO} /{ }^{13} \mathrm{CO} 1-0$ line intensity ratio $(>30$ Aalto et al. 1991) implies a low to moderate optical depth of the ${ }^{12} \mathrm{CO}$ line. Together with subthermally excited low- $J$ CO lines, this suggests the presence of a large filling factor of diffuse molecular gas (Aalto et al. 1995). The concept of diffuse gas in ultraluminous galaxies - among them Arp 220 - was extensively modelled and discussed by Downes \& Solomon (1998).

For Mrk 231, a similar diffuse gas phase may be suggested by the large $\mathrm{CO} /{ }^{13} \mathrm{CO} 2-1$ line intensity ratio (Glenn \& Hunter 2001) although further observations and radiative transfer modeling is necessary. For NGC 4418, the presence of diffuse molecular gas is unexplored and multi-wavelength $\mathrm{CO}$ and ${ }^{13} \mathrm{CO}$ modeling is required to address this issue.

\subsection{XDR chemistry}

The X-ray irradiation of molecular gas leads to a so-called X-ray dominated region (e.g., Maloney et al. 1996; Lepp \& Dalgarno 1996) similar to PDRs associated with bright UV sources (Tielens \& Hollenbach 1985). The more energetic $(1-100 \mathrm{keV})$ $\mathrm{X}$-ray photons penetrate large columns $\left(10^{22}-10^{24} \mathrm{~cm}^{-2}\right)$ of gas and lead to a different ion-molecule chemistry. Species like $\mathrm{C}$, $\mathrm{C}^{+}$and $\mathrm{CO}$ co-exist (unlike the stratified PDR) and the abundances of $\mathrm{H}^{+}$and $\mathrm{He}^{+}$are much larger. Molecules like $\mathrm{H}_{2} \mathrm{O}$ and $\mathrm{OH}$ can be formed more efficiently because molecular gas resides at higher temperatures, a consequence of the fact that ionization heating (rather than photo-electric heating in PDRs) dominates and is more efficient (about 70\%). Models of XDRs by Meijerink \& Spaans (2005) indicate that the HNC/HCN column density ratio is elevated, and reaches a value of $\sim 2$, for gas densities around $10^{5} \mathrm{~cm}^{-3}$. This while PDRs and quiescent cloud regions exhibit ratios of unity or less. Hence, ULIRGS that contain an AGN and possess high gas densities are likely candidates for overluminous HNC emission (see Meijerink et al. 2007, for details).

In order to compare with observations Fig. 6 presents a grid of one dimensional single-sided PDR/XDR slab models in density and $\mathrm{FUV} / \mathrm{X}$-ray irradiation for $\mathrm{HNC} / \mathrm{HCN} 3-2$ line ratios (Meijerink et al. 2007). The model clouds have a size of $1 \mathrm{pc}$, but we have verified that our choice of cloud size does not influence the computed line intensity ratios. We have assumed that an individual cloud in a galaxy has a velocity line width of $5 \mathrm{~km} \mathrm{~s}^{-1}$. The impinging X-ray flux follows a -0.9 powerlaw in energy between 1 and $100 \mathrm{keV}$, typical of a Seyfert nucleus. Recall here that the total flux in the Habing field (between 6 and $13.6 \mathrm{eV}$ ) is $1.6 \times 10^{-3} \mathrm{erg} \mathrm{s}^{-1} \mathrm{~cm}^{-2}$. Hence, compared to a PDR, we consider enhancement factors of the ambient radiation field of $10^{3}-10^{5}$, typical of gas exposed to an AGN (Maloney et al. 1996; Meijerink \& Spaans 2005). HCN and HNC are formed in equal amounts through dissociative recombination of $\mathrm{HCNH}^{+}$ (branching ratio 50-50). In XDRs, the degree of ionization is much larger than in PDRs. Hence, reactions with, e.g., $\mathrm{He}^{+}, \mathrm{H}^{+}$, $\mathrm{C}^{+}$and many others, that lead to the formation and destruction of $\mathrm{HCN}$ and $\mathrm{HNC}$ in an asymmetric way (with $\mathrm{HCNH}^{+}$and $\mathrm{H}_{2} \mathrm{NC}^{+}$ as intermediates), are much more important. $\mathrm{HNC}$ is particularly favored over HCN for moderate, 1-10 erg s $\mathrm{cm}^{-1} \mathrm{~cm}^{-2}$, values of the impinging flux, at an ambient density of $n \geq 10^{5} \mathrm{~cm}^{-3}$, where the abundance of ions exceeds that in a PDR by about an order of magnitude (Meijerink et al. 2006, their Fig. 1). Note that the different abundance gradients that occur for HCN and HNC (Meijerink \& Spaans 2005, their Fig. 10) at least provides a necessary condition for the HCN and HNC line intensity profiles to differ, i.e., to sample different kinematic regions in the nucleus of an active galaxy.

In PDRs, the ionization degree is modest and set by the ambient cosmic ray ionization rate. Consequently, the $\mathrm{HNC} / \mathrm{HCN}$ emission line ratios level off to unity at large, $>10^{22} \mathrm{~cm}^{-2}$, depths into the cloud. Note that a strongly elevated cosmic ray ionization rate is expected in systems that exhibit a large star formation rate, like the $250 M_{\odot} \mathrm{yr}^{-1}$ for Arp 220, because the supernova rate will scale up proportionally for a Salpeter IMF. The models of Meijerink et al. (2006) show that even a cosmic ray rate of $5 \times 10^{-15} \mathrm{~s}^{-1}$ (roughly $200 M_{\odot} \mathrm{yr}^{-1}$ ) does not allow cosmic ray boosted PDRs to behave as genuine XDRs in terms of the $\mathrm{HNC} / \mathrm{HCN}$ ratio. This is basically because the very high energies of cosmic rays render their radiative interactions (cross sections scale as $1 / E^{3}$ ) with atoms and molecules rather weak.

A top-heavy (non-Salpeter) IMF would lead to a higher ionization rate for a fiducial star formation rate of $200 M_{\odot} \mathrm{yr}^{-1}$. Still, even for ionization rates as large as $3 \times 10^{-14} \mathrm{~s}^{-1}$, which would accommodate many extreme IMFs, cosmic-ray enhanced PDRs would still not mimic XDRs in terms of HNC, HCN and $\mathrm{HCO}^{+}$. In particular, $\mathrm{HNC} / \mathrm{HCN}$ would still be limited to unity for the very high cosmic ray ionization rate mentioned above. The reason is that X-rays couple much better to the gas than cosmic rays, i.e. their energies are lower, while large columns of gas can still be penetrated by them (unlike by UV photons), 
leading to an active ion-molecule chemistry that favours HNC and $\mathrm{HCO}^{+}$.

Finally, we note that the maximum $\mathrm{HNC} / \mathrm{HCN} 3-2$ line ratio of 1.6 in our model clouds is smaller than the abundance ratio of about 2 found by Meijerink \& Spaans (2005). This is a consequence of optical depth effects in the lines, i.e., one typically reaches a $\tau=1$ surface before the full $\mathrm{HNC} / \mathrm{HCN}$ abundance gradient is sampled. A larger line width than $5 \mathrm{~km} \mathrm{~s}^{-1}$ for individual model clouds would alleviate this.

\section{Discussion}

With existing data it will be difficult to distinguish between the two proposed scenarios of IR-pumping and XDRs. The reason for this is that the densities required for HNC abundance enhancements in an XDR are high, $n>10^{5} \mathrm{~cm}^{-3}$ (see previous section), resulting in a high excitation of the molecule. This is also the case for the IR-pumping, since the radiative excitation helps populating higher $J$-levels.

\subsection{A pumping scenario: observational confirmation}

The mid-IR and dust properties of the three observed galaxies are summarized in Table 4 and the requirements for pumping of HNC, discussed in 3.3, appear fulfilled in the nuclei of the galaxies. Below is a list of useful observational tests for IR pumping:

- Line ratios and excitation. If the $\mathrm{HNC} / \mathrm{HCN}$ line intensity ratio exceeds the theoretical abundance limit of 2 from an XDR then it is necessary to invoke radiative pumping to explain the observed line intensity ratio. This is for instance the case for NGC 4418 (see Sect. 4.3.2.) Furthermore, a significant difference in excitation between $\mathrm{HCN}$ and $\mathrm{HNC}$ would be a strong indication of radiative excitation of $\mathrm{HNC}$, for instance if the higher transition HNC lines are more luminous than those of HCN. Both molecules have similar critical densities, so a more excited HNC may well indicate radiative pumping.

- Vibrational absorption and emission lines. Searching for the $21.5 \mu \mathrm{m}$ absorption line that occurs when the HNC molecule absorbs the mid-IR continuum is a potential useful test. A possible caveat, however, is that the absorption lines may become filled-in by emission at similar wavelengths when the molecule falls back into the $v=0$ state. It is also possible to search for rotational transitions of vibrationally excited $\mathrm{HNC}$ in the millimeter/submillimeter range. They are, however, often close enough in frequency to the rotational bands that they become blended with them (they are also weaker than the purely rotational bands) therefore sensitive, high spectral resolution observations are required. Vibrationally excited HNC has been detected in the Galaxy towards the proto-planetary nebula CRL 618. The lines are emerging in the hot PDR between the inner H II region and the molecular envelope (Schilke et al. 2002).

- High resolution studies. High resolution, interferometric studies of HCN and HNC nuclear line emission will show the relative distribution and brightness of the two species. Mid-IR observations will reveal how the infrared continuum is distributed compared to the molecular line emission. Multi-transition observations of other high density tracer molecules at high resolution will provide an independent density measurement of the nuclear gas. One must select molecules that are unlikely to be affected by radiative excitation (such as CS).

\subsection{An XDR scenario: observational confirmation}

An $\mathrm{HNC} / \mathrm{HCN}$ ratio larger than unity may instead be an abundance effect caused by the presence of an XDR - rather than a PDR combined with radiative excitation (Meijerink \& Spaans 2005; Meijerink et al. 2007). The ambient densities, $10^{5} \mathrm{~cm}^{-3}$, and X-ray fluxes, $1-10 \mathrm{erg} \mathrm{s}^{-1} \mathrm{~cm}^{-2}$, that are required for the XDR interpretation of the Arp 220, NGC 4418 and Mrk 231 data are quite reasonable, and only require the presence of an AGN and an absorbing hydrogen column of $N_{\mathrm{H}}>10^{22.5} \mathrm{~cm}^{-2}$. However, the alternative of IR pumping cannot be excluded on these grounds. Below follows a list of observational tests for an XDR:

- $\mathrm{HCN} / \mathrm{HCO}^{+}$: one can look for modest, $<1, \mathrm{HCN} / \mathrm{HCO}^{+} 1-0$, $2-1$, etc. line ratios. The $\mathrm{HCO}^{+}$abundance is expected to increase more in XDRs than the HCN abundance and this leads to $\mathrm{HCN} / \mathrm{HCO}^{+}$rotational emission line ratios that are less than unity, as follows. A high X-ray flux leads to more destruction of $\mathrm{HCO}^{+}$at the edge of an XDR, through dissociative recombination, but the large degree of ionization, and the associated ion-molecule chemistry, more than compensates for this at hydrogen columns in excess of $10^{23} \mathrm{~cm}^{-2}$ and/or flux-to-density ratios of less than $10^{-3} \mathrm{erg} \mathrm{s}^{-1} \mathrm{~cm}$ (Meijerink $\&$ Spaans 2005). That is, penetrating $\mathrm{X}$-rays, much like cosmic rays, maintain a large abundance of $\mathrm{H}_{3}^{+}$. This ion reacts with $\mathrm{O}$ to form $\mathrm{OH}^{+}$and leads to $\mathrm{H}_{3} \mathrm{O}^{+}$through reactions with $\mathrm{H}_{2}$. Dissociative recombination then leads to $\mathrm{OH}$ (and water) which can react with $\mathrm{C}^{+}$to form $\mathrm{CO}^{+}$(and $\mathrm{HCO}^{+}$), subsequent reaction with $\mathrm{H}_{2}$ leads to $\mathrm{HCO}^{+}$. Additional pathways exist where $\mathrm{OH}$, and $\mathrm{H}_{2} \mathrm{O}$, form directly through endoergic neutral-neutral reactions $\mathrm{O}+\mathrm{H}_{2}$ and $\mathrm{OH}+\mathrm{H}_{2}$ followed by reaction with $\mathrm{C}^{+}$. Also, once $\mathrm{CO}$ is abundant, reaction with $\mathrm{H}_{3}^{+}$leads to $\mathrm{HCO}^{+}$directly. Note in this that the often cited Lepp \& Dalgarno (1996) XDR work clearly shows that $\mathrm{HCO}^{+}$enjoys large abundances over a wider range of ionization rates than $\mathrm{HCN}$ does, favoring $\mathrm{HCO}^{+}$over $\mathrm{HCN}$ when a strong source of ionization plays a role. One may wonder about the influence of cosmic rays, as a source of ionization, on the structure of PDRs.

Model 3 of Jansen et al. (1995) shows that raising the cosmic ray ionization rate by a factor of 4 over the Galactic value, and thus boosting the level of ions, enhances the $\mathrm{HCO}^{+}$abundance by more than a factor of three, while $\mathrm{HCN}$ and HNC benefit only on the factor of two level. Such a high cosmic ray rate PDR behaves somewhat like an XDR, but is less extreme $\left(\mathrm{HCN} / \mathrm{HCO}^{+} \sim 1\right.$ or a bit less), as discussed in Meijerink et al. (2006). The latter authors also show that a regular PDR with a low cosmic ray ionization rate, which would apply when no supernovae have gone off yet, leads to $\mathrm{HCN} / \mathrm{HCO}^{+}>1$ for densities of $\sim 10^{5} \mathrm{~cm}^{-3}$ and up.

Thus, one can make a clear comparison between low cosmic ray rate PDRs and XDRs if the PDR component does not occupy a larger area in the beam. The XDR/AGN contribution loses to the star formation/FUV signal if the latter is more spread out and care should therefore be taken with the interpretation of correlations between nuclear activity and elevated $\mathrm{HCN} / \mathrm{HCO}^{+}$ratios.

Gracia-Carpio et al. (2006) find elevated $\mathrm{HCN} / \mathrm{HCO}^{+}$ $1-0$ line ratios in ULIRGs - and in general they find that the line ratio correlates with FIR luminosity. They attribute this to the presence of an AGN where the X-rays affect the chemistry to impact the $\mathrm{HCN} / \mathrm{HCO}^{+}$abundance ratio. However, as we discuss in this paper, for dense XDRs $\left(n>10^{5} \mathrm{~cm}^{-3}\right)$ one would not expect the $\mathrm{HCN}$ to be more abundant than $\mathrm{HCO}^{+}$. 
It is possible that the findings of Gracia-Carpio et al. can be explained by excitation effects (e.g. IR-pumping) or by other chemical processes affecting abundances.

- Very high J CO emission: unambiguous diagnostics are provided by highly excited $(J>10)$ rotational lines of $\mathrm{CO}$ (Meijerink et al. 2007). XDRs more easily produce very warm CO compared to PDRs (also PDRs with high cosmic ray rates). This is because $\mathrm{CO}$ is present already at the warm XDR edge, initiated through $\mathrm{C}^{+}+\mathrm{OH}$ route above, while the higher UV flux at the edge of PDRs, for the same density and total energy input, suppresses $\mathrm{CO}$. These high $J$ CO lines will be accessible to the HIFI instrument on Herschel - and to instruments on SOFIA - and can already be observed in absorption in the near-infrared with Subaru. A combination of overluminous HNC emission and very high $J$ CO emission (or absorption) would be compelling evidence for an XDR/AGN.

\subsection{Pumping or XDRs in Arp 220, NGC 4418, Mrk 231}

Arp 220 is an ultraluminous gas-rich merger of two galaxies where the two nuclei are separated by $\sim 300$ pc (Graham et al. 1990). The nature of the nuclear power source of Arp 220 is yet not established (see for instance Sakamoto et al. 1999, Sect. 7 for a discussion) since the activity is deeply enshrouded by dust. Regardless of whether the intense infrared radiation is driven by the nuclear starburst alone - or whether it is boosted by an AGN - the mid-IR properties of Arp 220 fulfill the criteria for $21.5 \mu \mathrm{m}$ HNC pumping (see Table 4). Sakamoto et al. (1999) find that a substantial fraction of the molecular gas can be found in compact, rotating nuclear structures, which should be closely associated with the mid-infrared sources. Even though intense emission from molecules such as $\mathrm{CS}, \mathrm{CN}, \mathrm{HCO}^{+} 1-0, \mathrm{HCN}$ and $\mathrm{HC}_{3} \mathrm{~N}$ (Radford et al. 1990; Solomon et al. 1992), Wiedner et al. 2004, APCH02 indicate gas densities in excess of $10^{4} \mathrm{~cm}^{-3}$, the brightness temperature of the mid-IR field is large enough to compete with collisions at densities up to at least $10^{5} \mathrm{~cm}^{-3}$. The high excitation of $\mathrm{HNC}$, as compared to $\mathrm{HCN}$, is an expected pumping-signature since it should result in elevated excitation temperatures (also of the higher transitions) compared to those expected from typical collisional excitation.

For Arp 220, the observed $H N C / H C N$ 3-2 line ratio of 1.9 is close to the theoretical maximum of 2 for the abundance ratio in an XDR (Sect. 3.4) and all of the HNC and HCN 3-2 emission must emerge from an XDR with optically thin emission for an XDR to suffice in explaining the line ratio. Thus, we propose that the HNC emission is affected by pumping in Arp 220 with the possible additional effect of an XDR. This would require Arp 220 to host a buried AGN, but such an AGN component does not have to be energetically dominant.

Pumping of HCN?: it is also possible that part of the $\mathrm{HCN}$ emission is affected by radiative excitation as well, but to a lesser degree. The requirements for the $14 \mu \mathrm{m}$ field to be intense enough to compete with collisions for the HCN molecule are greater for $\mathrm{HCN}$ than the requirements to pump HNC and are fulfilled in a smaller region of the western nucleus of Arp 220. Barvainis et al. (1997) suggested that the excitation of the HCN emission of the Cloverleaf Quasar may be affected by absorption of $14 \mu \mathrm{m}$ continuum. Clearly, the excitation of HCN in Arp 220 warrants further study.

Even though NGC 4418 is far from being an ultraluminous galaxy, several of its mid-IR and molecular properties appear remarkably similar to those of Arp 220 (Table 4). Both galaxies exhibit rich molecular chemistry similar to that of a Galactic hot core (e.g., APCH02, Monje \& Aalto, in preparation; Martin-Pintado et al., in preparation). The far-infrared surface brightness is estimated to be extremely high (Evans et al. 2003) and most of its molecular gas is concentrated in the inner kpc (Dale et al. 2005). It is unclear what is driving the IR luminosity of NGC 4418. Imanishi et al. (2004) report that the estimated star formation luminosity from the observed PAH emission can account for only a small fraction of the infrared luminosity thus suggesting that NGC 4418 may be largely AGN-driven. They furthermore report an elevated $\mathrm{HCN} / \mathrm{HCO}^{+} 1-0$ line intensity ratio which they suggest is due to the presence of an AGN-powered XDR-chemistry, although depth-dependent models by Meijerink \& Spaans (2005) and Meijerink et al. (2006) seem to suggest an opposite trend, with $\mathrm{HCN} / \mathrm{HCO}^{+} 1-0<1$ under X-ray irradiation (see Sect. 4.2.1). Properties presented in Table 4 show that conditions for nuclear IR pumping of HNC are fulfilled. The HNC 3-2/1-0 line ratio is not globally superthermal - as is the case for Arp 220 but HNC is significantly more highly excited than $\mathrm{HCN}$.

For NGC 4418, the observed $\mathrm{HNC} / \mathrm{HCN} 3-2$ line ratio of 2.3 exceeds the theoretical maximum of 2 for the abundance ratio in an XDR (Sect. 3.4) and we conclude that mid-IR pumping is necessary to explain the observed $H N C / H C N$ 3-2 line ratio. However, an additional effect from an XDR is likely and should be investigated with the proposed observational methods.

Mrk 231 is an ultraluminous Seyfert 1 galaxy with a powerful starburst surrounding the AGN. Davies et al. (2005) have studied the starburst and found a warped starburst disk of dimensions $200 \mathrm{pc}$ - with a high stellar velocity dispersion of $100 \mathrm{~km} \mathrm{~s}^{-1}$ (recent results indicate that velocity dispersions of ULIRGs may in general be even higher (Dasyra et al. 2006)). Vibrationally excited $\mathrm{H}_{2}$ lines are found throughout the starburst disk and the overall ISM properties of the disk must be highly unusual. The presence of an AGN driving the Seyfert 1 characteristics is well established in Mrk 231 - in contrast to the other two galaxies in the sample. However, Mrk 231 is not as clear a case for HNC overluminosity as Arp 220 or NGC 4418 - even though nuclear conditions should be favourable for pumping as indicated in Table 4. The HNC 3-2 luminosity is a factor of 1.5 greater than the HCN 3-2 luminosity - but this is largely due to a difference in line width, not peak brightness as is the case for Arp 220 and NGC 4418. The HCN line width agrees with that of ${ }^{12} \mathrm{CO} 2-1$ and is consistent with the rotational velocities expected from the near-face-on 1 kpc disk. The HNC 3-2 (and HNC 4-3) line width is, however, almost a factor of two greater than that of $\mathrm{HCN}$ and ${ }^{12} \mathrm{CO}$. The HNC 3-2 line peaks at the same velocity as the $\mathrm{OH}$ megamaser emission (e.g. Richards et al. 2005) and has a similar line width. This may imply that the HNC 3-2 emission is more nuclear than the HCN (although we require high resolution information to confirm this). In this scenario there will be a radial dependence in the intensity ratio, where the $\mathrm{HNC} / \mathrm{HCN}$ line intensity ratio may be close to unity in the disk, and then increases towards the nucleus.

$\mathrm{HCN}$ is subthermally excited, suggesting overall densities between $10^{4}$ and $10^{5} \mathrm{~cm}^{-3}$ and the gas kinetic temperatures are high as suggested by the interferometric observations (see Table 4). HNC is more highly excited, suggesting it is emerging from higher density or warmer gas - or that it is affected by radiative excitation. Both these suggestions are consistent with $\mathrm{HNC}$ being more centrally concentrated than $\mathrm{HCN}$.

The observed $\mathrm{HNC/HCN}$ 3-2 line ratio of 1.5 is less than the theoretical maximum of 2 (Sect. 3.4) for an XDR and with existing data we cannot distinguish between pumping and X-ray chemistry as the main source behind the overluminosity of $H N C$. 


\subsection{The extreme nuclear ISM}

Regardless of whether it is mid-IR pumping or X-ray induced chemistry (or a combination of both) that is behind the overluminous HNC 3-2 emission, it is clear that the prevailing ISM conditions are unusual compared to similar scales in more normal galaxies. The buried nuclear activity affects the properties of the ISM on scales large enough to create global effects on observed molecular line ratios.

When comparing the HNC 1-0 luminosities of more nearby galaxies studied by Hüttemeister et al. (1995) APCH02 find that the relative HNC 1-0 luminosity appeared to increase with luminosity - even if this effect is small. In general, normal nearby galaxies have $\mathrm{HNC} / \mathrm{HCN} 1-0$ line ratios well below unity. Both $\mathrm{APCH} 2$ and Hüttemeister et al. find that $\mathrm{HNC} / \mathrm{HCN} 1-0$ line ratios of unity (or exceeding unity) are rare.

Both Arp 220 and NGC 4418 show deep silicate absorption in their mid-IR spectra (e.g., Spoon et al. 2001) suggesting visual extinction exceeding $50 \mathrm{mag}$. It is interesting to note that it is these two galaxies that show the greatest overluminosity in $\mathrm{HNC}$ - and not the Seyfert 1 galaxy Mrk 231. It is tempting to suggest that a deeply buried, compact and highly intense source heats the large masses of dust surrounding the nuclei of Arp 220 and NGC 4418 and that the re-radiated IR emission is pumping the HNC emission. It is quite possible that we have nuclear XDRs in NGC 4418 and in Arp 220, but we note that their effects are likely boosted by mid-IR pumping since the observed $\mathrm{HNC} / \mathrm{HCN} 3-2$ line ratios exceed the theoretical maximum of 2 for the $\mathrm{HNC} / \mathrm{HCN}$ abundance ratio from an XDR.

Future, high resolution mm observations, and further studies in the mid-IR and high energy X-rays will help to reveal which one of the two suggested mechanisms is the dominant one behind the overluminous HNC 3-2 emission.

\section{Conclusions}

1. We have detected surprisingly bright HNC 3-2 emission in the ultraluminous galaxies Arp 220 and Mrk 231 and in the IR luminous galaxy NGC 4418. In all three cases the HNC 3-2 emission outshines the HCN 3-2 emission by factors 1.5-2.3. We also detected HNC 4-3 emission in Mrk 231 which shows that HNC line emission continues to be bright also at higher transitions.

2. We propose that the HNC emission is pumped by $21.5 \mu \mathrm{m}$ IR continuum emission via its degenerate bending mode. This means that the emission is no longer dominated by collisions and its luminosity may not be used to deduce information on gas density. Ways to test for pumping include high resolution studies, searches for the mid-IR absorptions lines and the rotational-vibrational HNC lines.

3. Alternatively, X-rays produced by an embedded AGN can influence the chemistry in favor of $\mathrm{HNC}$, with $\mathrm{HNC} / \mathrm{HCN}$ emission line ratios of $1-2$. This may produce an overluminosity of $\mathrm{HNC}$ without the effects of pumping. Small $\mathrm{HCN} / \mathrm{HCO}^{+}$line intensity ratios and, in particular, the detection of very highly excited $\mathrm{CO}(J \geq 10)$ would confirm the XDR interpretation of the overluminous HNC emission in Arp 220, Mrk 231 and NGC 4418. For NGC 4418 however, the observed $\mathrm{HNC} / \mathrm{HCN} 3-2$ line ratio exceeds the theoretical maximum for XDR chemistry, and it is necessary to invoke mid-IR pumping to explain the observed line ratios.

4. We conclude that for all three galaxies the molecular ISM is in an extreme state - where the the physical conditions and/or chemistry of the gas is heavily influenced by the radiation field from the central activity. The ISM properties are very different from those of typical Galactic starforming molecular clouds.

Acknowledgements. Many thanks to the JCMT and IRAM staff for their support during observations. Molecular databases that have been helpful include The Cologne Database and the molecular database of Crovoisier. We are grateful for discussions with J. Black, A. E. H. Olofsson and R. Meijerink, and for help with observations by E. Olsson, R. Monje and K. Torstensson. Our most heartfelt thanks to D. Lis of Caltech who helped us find and extract the Mrk $231 \mathrm{HCN} 3-2$ data from the CSO.

\section{References}

Aalto, S. 2005, in The Neutral ISM of Starburst Galaxies, ed. S. Aalto, S. Huttemeister, \& A. Pedlar, ASP, 320, 1

Aalto, S., Johansson, L. E. B., Booth, R. S., \& Black, J. H. 1991, 249, 323

Aalto, S., Booth, R., \& Black, J. H. 1995, A\&A, 300, 369

Aalto, S., Polatidis, A. G., Hüttemeister, S., \& Curran, S. J. 2002, A\&A, 381, 783 (APHC02)

Barvainis, R., Maloney, P., Antonucci, R., \& Alloin, D. 1997, ApJ, 484, 695

Boger, G. I., \& Sternberg, A. 2005, ApJ, 632, 302

Boger, G. I., \& Sternberg, A. 2006, ApJ, 645, 314

Bryant, P. M., \& Scoville, N. Z. 1999, AJ, 117, 2632

Cernicharo, J., Pardo, J. R., \& Weiss, A. 2006, ApJ, 646, L49

Curran, S. J., Aalto, S., \& Booth, R. S. 2000, A\&AS, 141, 193

Dale, D. A., Sheth, K., Helou, G., Regan, M. W., \& Hüttemeister, S. 2005, AJ, 129,2197

Dasyra, K. M., Tacconi, L. J., Davies, R. I., et al. 2006, ApJ, 638, 745

Davies, R. I., Tacconi, L. J., \& Genzel, R. 2004, ApJ, 613, 781

Downes, D., \& Solomon, P. M. 1998, ApJ, 507, 615

Evans, A. S., Becklin, E. E., Scoville, N. Z., et al. 2003, AJ, 125, 2341

Herpin, F., \& Cernicharo, J. 2000, ApJ, 530, L129

Gao, Y., \& Solomon, P. M. 2004, ApJS, 152, 63

González-Alfonso, E., Smith, H. A., Fischer, J., \& Cernicharo, J. 2004, ApJ, 613, 247

Glenn, J., \& Hunter, T. R. 2001, ApJS, 135, 177

Graham, J. R., Carico, D. P., Matthews, K., et al. 1990, ApJ, 354, L5

Helfer, T. T., \& Blitz, L. 1993, ApJ, 419, 86

Hirota, T., Yamamoto, S., Mikami, H., \& Ohishi, M. 1998, ApJ, 503, 717

Hüttemeister, S., \& Aalto 2001, in The Central Kiloparsec of Starbursts and AGN, ed. J. H. Knapen, J. E. Beckman, I. Shlosman, \& T. J. Mahoney, 619

Hüttemeister, S., Henkel, C., Mauersberger, R., et al. 1995, A\&A, 295, 571 Imanishi, M., Nakanishi, K., Kuno, N., \& Kohno, K. 2004, AJ, 128, 2037 Jansen, D. J., van Dishoeck, E. F., Black, J. H., Spaans, M., \& Sosin, C. 1995, A\&A, 302, 223

Lepp, S., \& Dalgarno, A. 1996, A\&A, 306, L21

Maloney, P. R., Hollenbach, D. J., \& Tielens, A. G. G. M. 1996, ApJ, 466, 561

Meijerink, R., \& Spaans, M. 2005, A\&A, 436, 397

Meijerink, R., Spaans, M., \& Israel, F. P. 2006, ApJ, 650, L103

Meijerink, R., Spaans, M., \& Israel, F. P. 2007, A\&A, 461, 793

Radford, S. J. E., Delannoy, J., Downes, D., et al. 1991, in Dynamics of Galaxies and Their Molecular Cloud Distribution (Kluwer Academic Publishers), ed. F. Combes, \& F. Casoli, IAU Symp., 146, 303

Richards, A. M. S., Knapen, J. H., Yates, J. A., et al. 2005, MNRAS, 364, 353

Roche, P. F., Aitken, D. K., Smith, C. H., \& James, S. D. 1986, MNRAS, 218 , 19

Sakamoto, K., Scoville, N. Z., Yun, M. S., et al. 1999, ApJ, 514, 68

Schilke, P., Walmsley, C. M., Pineau de Forest, G., et al. 1992, A\&A, 256, 595 (S92)

Schilke, P., Comito, C., \& Thorwirth, S. 2002, ApJ, 582, 101

Scoville, N. Z., Yun, M. S, \& Bryant, P. M. 1997, ApJ, 484, 702

Soifer, B. T., Neugebauer, G., Matthews, K., et al. 1999, ApJ, 513, 207

Soifer, B. T, Neugebauer, G., Matthews, K., et al. 2000, AJ, 119, 509

Solomon, P. M., Downes, D., \& Radford, S. J. E. 1992, ApJ, 387, L55

Spoon, H. W. W., Keane, J. V., Tielens, A. G. G. M., Lutz, D., \& Moorwood, A. F. M. 2001, A\&A, 365, 353

Tielens, A. G. G. M., \& Hollenbach, D. 1985, ApJ, 291, 722

Wiedner, M., Wilson, C., Reid, M., Saito, M., \& Menten, K. 2004, in The Neutral ISM in Starburst Galaxies, ed. S. Aalto, S. Huttemeister, \& A. Pedlar, ASP, 320,35 